\title{
Neuronal Ceroid Lipofuscinosis Type 2
}

National Cancer Institute

\section{Source}

National Cancer Institute. Neuronal Ceroid Lipofuscinosis Type 2. NCI Thesaurus. Code C85864.

A condition associated with mutation(s) in the TPP1 gene, encoding tripeptidyl-peptidase-

1. The condition is one of a group of genetically heterogeneous neurodegenerative disorders, characterized by accumulation of intracellular lipopigments. 